\title{
Generating a New Hyperchaotic Pan System Via State Feedback Control
}

\section{Saad Fawzi AL-Azzawi}

Department of Mathematics, College of Computer Sciences and Mathematics, University of Mosul, Mosul, Iraq E-mail address: saad_fawzi78@yahoo.com

Received on: $24 / 04 / 2012$

Accepted on: 28/06/2012

This paper proposes a new four-dimensional continuous autonomous hyperchaotic system based on the 3D Pan system by introducing a nonlinear state feedback controller. Dynamical behaviors of the new system are analyzed, both theoretically and numerically, including equilibrium points, Lyapunov exponents spectrum stability and bifurcation, finally, an illustrative example is given.

Keywords: Hyperchaos, Feedback control, Lyapunov exponent, Pan system, Stability.

$$
\begin{aligned}
& \text { توليد نظام Pan كثير الاضطراب جليد من خلال سيطرة حالة التغذية العكسية } \\
& \text { الملخص } \\
& \text { في هذا البحث تم اقترح نظام جديد رباعي الإبعاد مستمر مستقل ذاتيا كثير الاضطراب معتمدا على نظام } \\
& \text { ثلاثي الأبعاد بتقديم سيطرة حالة التغذية العكسية غير الخطية. وتم تحليل السلوك الحركي للنظامِ الجديدِ }
\end{aligned}
$$

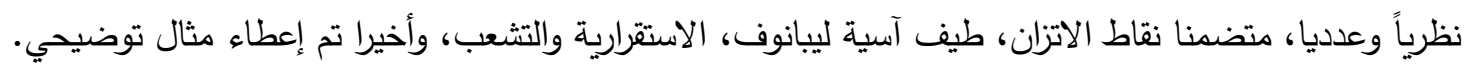

$$
\begin{aligned}
& \text { الكلمات المفتاحية: شديد الاضطراب, سيطرة التغذية , اسية لايبنوف , نظام Pan الاستقرارية. }
\end{aligned}
$$

\section{Introduction:}

In 1963, Lorenz discovered the first chaotic system when he studied atmospheric convection, since then, the Lorenz system has been extensively studied in the field of chaos theory and dynamical systems [8]. In recent years, hyperchaos generation and control have been extensively studied due to its theoretical and practical applications in the fields of communications, laser, neural work, nonlinear circuit, mathematics, and so on $[6,8,10]$.

Historically, hyperchaos was firstly reported by Rössler. That is, the noted fourdimensional (4D) hyperchaotic Rössler system [6, 8], Hyperchaotic system is usually defined as a chaotic system with at least two positive exponents $[2,3,6,9,10]$ and is expanded in two or more directions, so the hyperchaotic system is more complicated than chaotic system $[3,9]$.

Over the last two decades, there are various hyperchaotic systems discovered in high-dimensional systems, Typical examples are four-dimensional (4D) hyperchaotic Rössler system, 4D hyperchaotic Lorenz-Haken system, 4D hyperchaotic Chua's circuit, and 4D hyperchaotic Chen system [2,6], very recently, hyperchaos was found numerically and experimentally by adding a simple state feedback controller $[6,8,10]$.

In 2006, Chen, Lu, Lü and Yu generated hyperchaotic Lü system by using a state feedback controller and studied the dynamical behaviors for this system [2]. At the same year, Wang, Zhen and Li present hyperchaotic Lorenz system and also studied the dynamical behaviors for this system [10]. In 2008, Wang modified another hyperchaotic Lorenz system which is different from system by Wang, Zhen and Li [7]. later more and more hyperchaotic systems are generated such as another hyperchaotic Lü system (2009) [3], hyperchaotic Liu system (2009) [8], four-wing hyperchaotic system (2010) [9], finally, hyperchaotic Pan system (2011) [5].

In this paper, a new four-dimension hyperchaotic Pan system is constructed based on a three-dimensional Pan system by introducing a nonlinear state feedback 
controller, and we investigated some basic properties and behaviors for this system numerically and analytically, including symmetry and invariance, dissipative and existence of attracter, equilibrium points, Lyapunov exponents spectrum, stability and bifurcation .

\section{2- System Description}

Pan system or L $\ddot{u}$-like system [5] is found by L. Pan, D. Xu and W. Zhou in 2010 which is similar to the Lorenz system, but it is not topological equivalent with the Lorenz system [4]. And this system provides another interesting framework for advanced control techniques since it is more complex than the Lorenz system and Chua system. Moreover, it is more difficult to control the Pan system than the other than those already known chaotic systems due to the rapid change of the velocity in the z-direction [4].

The mathematical model of Pan system is a system of nonlinear ordinary differential equations which has the form:

$$
\left.\begin{array}{l}
\dot{x}=a(y-x) \\
\dot{y}=c x-x z \\
\dot{z}=x y-b z
\end{array}\right\}
$$

in which $a, b$ and $c$ are real constants. when $a=10, b=2$, and $c=16$ the Lyapunov exponents by Wolf Algorithm are $L E_{1}=0.8311, L E_{2}=0.0000$ and $L E_{3}=-12.5113$. The Lyapunov exponents spectrum and Phase portraits of the system (1) are shown in Fig.1 and Fig.2 respectively [5].

The Lyapunov dimension of system (1) is given as follows [5]:

$$
D_{L E}=j+\frac{1}{\left|L E_{j+1}\right|} \sum_{i=1}^{j} L E_{i}=2+\frac{L E_{1}+L E_{2}}{\left|L E_{3}\right|}=2+\frac{0.8311+0}{|-12.5113|}=2.0664 \text {. }
$$

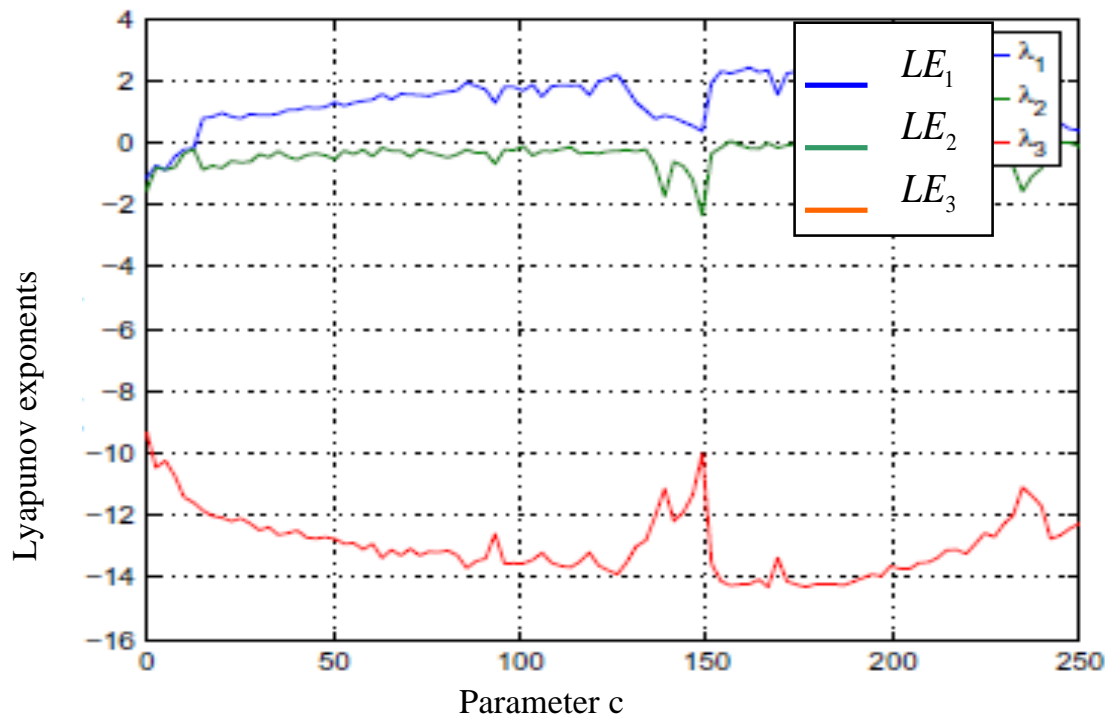

Fig. 1. Lyapunov exponents spectrum of Pan chaotic system versus parameter $\mathrm{c}$ 

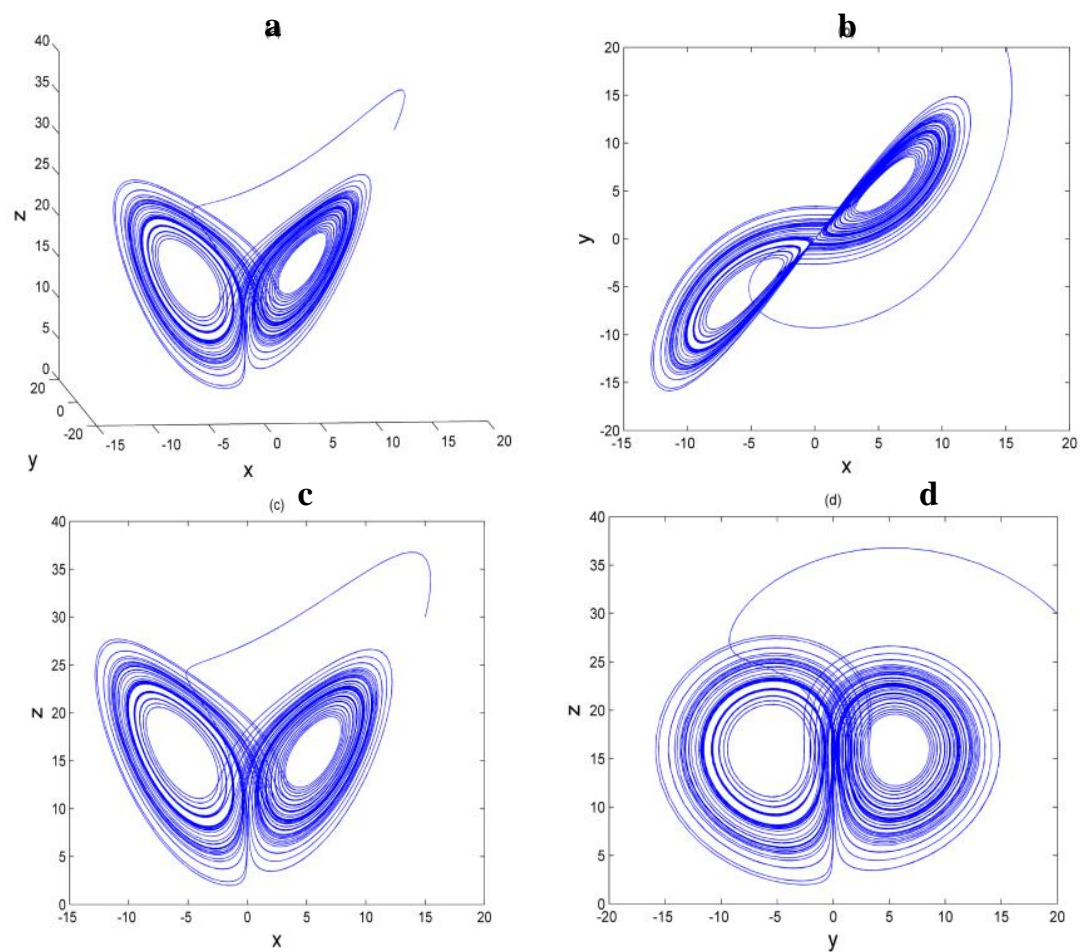

Fig. 2. Phase portraits of Pan system in (a) the $x-y-z$ space; and projected on (b) the $x-y$ plane; (c) the $\mathrm{x}-\mathrm{z}$ plane; and (d) the $\mathrm{y}-\mathrm{z}$ plane.

\section{3- Helping Results:}

The first two remarks give us information about the roots of a polynomial and can be applied to the characteristic polynomial of a matrix.

\section{Remark 1 (Coefficient Test) [1]:}

Suppose that:

$$
f(\lambda)=\lambda^{n}+A \lambda^{n-1}+B \lambda^{n-2}+C \lambda^{n-3}+\ldots \ldots . .+Y \lambda+Z
$$

where the coefficients are real. If any coefficient of $f(\lambda)$ is either zero or negative, then, at least, one root has a nonnegative real part.

\section{Remark 2 (Routh- Hurwitz Test) [1]:}

All the roots of the indicated polynomial have negative real parts precisely when the given conditions are met.

- $\lambda^{2}+A \lambda+B: A>0, B>o$

- $\lambda^{3}+A \lambda^{2}+B \lambda+C: A>0, C>0, A B-C>0$.

- $\lambda^{4}+A \lambda^{3}+B \lambda^{2}+C \lambda+D: A>0, A B-C>0,(A B-C) C-A^{2} D>0, D>0$.

\section{Remark 3 (Trace Test) [1]:}

Suppose that $A_{1} \mathrm{~s}$ an $n \times n$ matrix of real constants and that trace $A_{1}=a_{11}+a_{22}+\ldots \ldots . .+a_{n n}$ is negative (positive). Then, at least, one eigenvalue of $A_{1}$ has a negative (positive) real part. If the trace is zero, then either all eigenvalues have zero real parts, or there is a pair of eigenvalues whose real parts have opposite signs. 


\section{Proposition 1 [1]:}

1) The coefficient test does not apply to the polynomial with a positive coefficient.

2) The Routh - Hurwitz test applies to polynomials of degree no more than four.

3) The trace test applies directly to matrix, so there is no need to find the characteristic polynomial.

Finally, the Routh-Hurwitz test is the best from the coefficient test and trace test.

\section{Remark 4 (Generating Hyperchaos) $[2,5,6,7,8]$ :}

To generate hyperchaos from the dissipatively autonomously polynomial systems by using a state feedback controller, the state equation must satisfy the following two basic conditions:

The minimal dimension of the phase space of an autonomous system is at least four. The number of terms in the coupled equations giving rise to instability is at least two, of which, at least, one has a nonlinear function .

\section{Remark 5 (Lyapunov Exponents) [2, 6]:}

Assume that the Lyapunov exponents for a simple four dimension hyperchaotic system are $L E_{i}$ for $i=1,2,3,4$ satisfying $L E_{1}>L E_{2}>L E_{3}>L E_{4}$. Then, the dynamical behaviors of this system can be classified as follows:

(1) For $L E_{1}>L E_{2}>0, L E_{3}=0, L E_{4}<0$ and $L E_{1}+L E_{2}+L E_{4}<0$, system hyperchaos.

(2) For $L E_{1}>0, L E_{2}=0, L E_{4}<L E_{3}<0$ and $L E_{1}+L E_{3}+L E_{4}<0$, system chaos.

(3) For $L E_{1}=0, L E_{4}<L E_{3}<L E_{2}<0$, system has a periodic orbit.

(4) For $L E_{4}<L E_{3}<L E_{2}<L E_{1}<0$, system has an equilibrium point.

In the context of ordinary differential equations ODEs, the word "Bifurcation" has come to mean any marked change in the structure of the orbits of a system (usually nonlinear) as a parameter passes through a critical value [1].

\section{Remark 6 (Hopf Bifurcation) [9]:}

Any system has a Hopf bifurcation if the following conditions are satisfied:

1- The Jacobian matrix has two purely imaginary roots and no other roots with zero real parts.

$$
2-\left.\frac{d}{d \mu}(\operatorname{Re}(\lambda(\mu)))\right|_{\mu=\mu 0} \neq 0
$$

\section{Remark 7 (Generating Hyperchaotic Pan system) [5]:}

Hyperchaotic Pan system is generated by introducing an additional state $\mathrm{u}$ to the first equation of system (1). Then, we get the following four-dimensional hyperchaotic system:

$$
\left.\begin{array}{l}
\dot{x}=a(y-x)+u \\
\dot{y}=c x-x z \\
\dot{z}=x y-b z \\
\dot{u}=-x z+d u
\end{array}\right\}
$$

where $(x, y, z, u) \in R^{4}$, and $a, b, c, d \in R$ are constant parameters. This system is called hyperchaotic Pan system, when parameters $a=10, b=8 / 3, c=28$ and $d=1.3$, we 
calculate the Lyapunov exponents with the Wolf Algorithm. and the four Lyapunov exponents of the hyperchaotic system (7) are

$L E_{1}=0.7340, \quad L E_{2}=0.2492, L E_{3}=0.0000$, and $L E_{4}=-11.3437$. The Lyapunov exponents spectrum and Phase portraits of the system (7) are shown in Fig.3 and Fig.4 respectively [5].

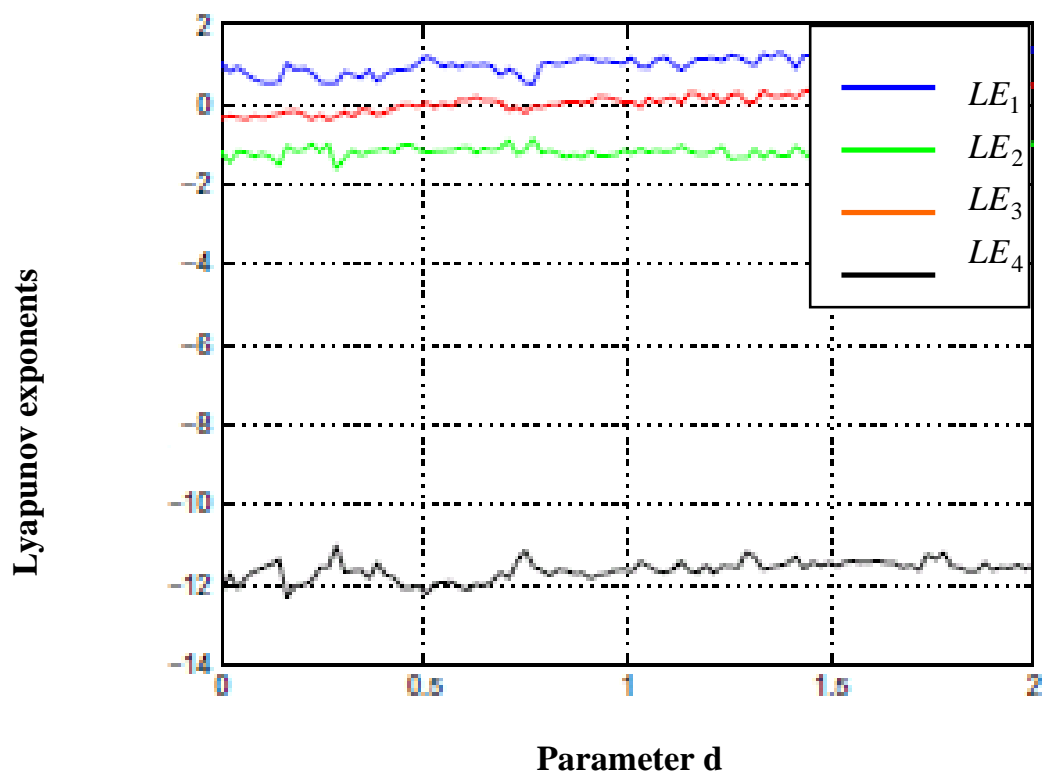

Fig. 3. Lyapunov exponents spectrum of Pan hyperchaotic system versus parameter $d$

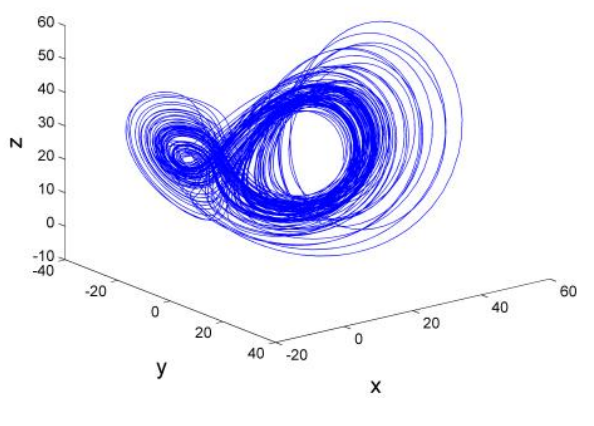

c

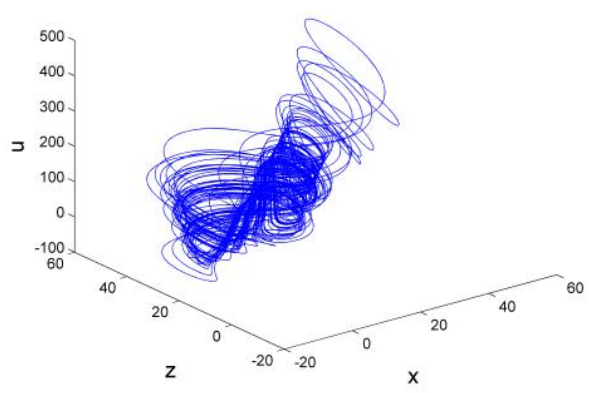

b

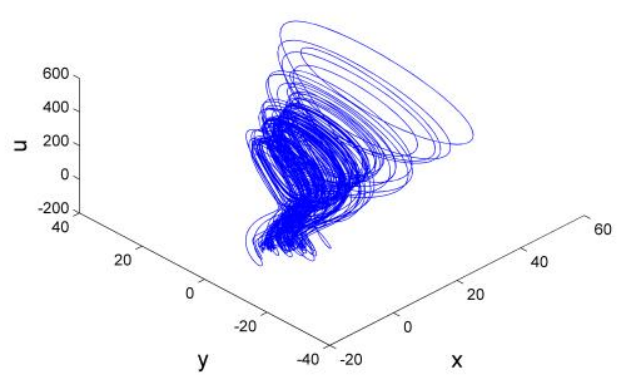

d

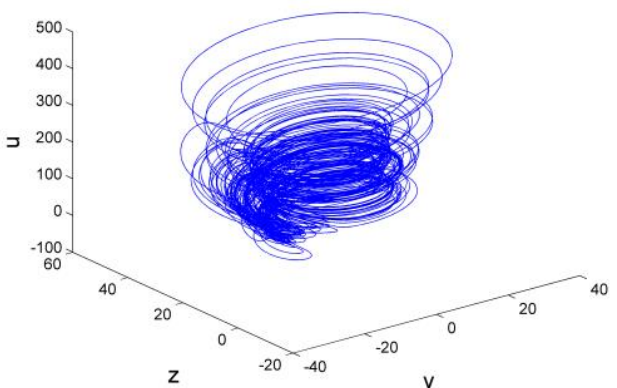

Fig. 4. Phase portraits of Pan hyperchaotic system in (a)the $x-y-z$ space;(b) the $x-y-u$ space, (c) the $\mathrm{x}-\mathrm{z}-\mathrm{u}$ space, and(d) the $\mathrm{y}-\mathrm{z}-\mathrm{u}$ space. 


\section{4- Main results:}

Based on Pan system and remark 4, we can construct a new four dimension hyperchaotic system by introducing a state feedback controller, as follows:

Add a nonlinear controller $u$ to the second equation of system (1), let $\dot{u}=x z+d y$, then we obtain a new hyperchaotic system

$$
\left.\begin{array}{l}
\dot{x}=a(y-x) \\
\dot{y}=c x-x z-u \\
\dot{z}=x y-b z \\
\dot{u}=x z+d y
\end{array}\right\}
$$

where $(x, y, z, u) \in R^{4}$, and $a, b, c, d \in R$ are constant parameters. For simplification, system (8) is called a new hyperchaotic Pan system in this paper.

In the following, we briefly describe some dynamical behaviors of the new hyperchaotic system (8).

\section{1- Symmetry:}

System (8) has a natural symmetry under the coordinates transformation $(x, y, z, u) \rightarrow(-x,-y, z,-u)$ which persists for all values of the system parameters. This means that system (8) is symmetric about the z-axis.

\section{2- Dissipative and existence of attracter:}

Obviously, from system (8), one has

$$
\operatorname{div} \vec{V}=\frac{\partial \dot{x}}{\partial x}+\frac{\partial \dot{y}}{\partial y}+\frac{\partial \dot{z}}{\partial z}+\frac{\partial \dot{u}}{\partial u}=-(a+b),
$$

Therefore, to make system( 8 ) be dissipative, it is required that $a+b>0$.

\section{3- Equilibrium Points:}

In order to obtain the equilibrium points of system (8), let $\dot{x}=\dot{y}=\dot{z}=\dot{u}=0$, and we can obtain the following expressions (10):

$$
\left.\begin{array}{l}
a(y-x)=0 \\
c x-x z-u=0 \\
x y-b z=0 \\
x z+d y=0
\end{array}\right\}
$$

From the above equations, we obtain three equilibrium points $O(0,0,0,0)$

$$
\begin{aligned}
& p_{1}(\sqrt{-b d}, \sqrt{-b d},-d, \sqrt{-b d}(c+d)), \\
& p_{2}(-\sqrt{-b d},-\sqrt{-b d},-d,-\sqrt{-b d}(c+d)) .
\end{aligned}
$$

when $-b d>0$ while if $-b d \leq 0$ then exists only one equilibrium point $O(0,0,0,0)$.

\section{4- Lyapunov Exponents and Lyapunov Dimension :}

We calculate the Lyapunov exponents for a new hyperchaotic system with the Wolf Algorithm by using MATLAB software, the numerical simulation was carried out with $a=10, b=8 / 3, c=2, d=0.2$, for initial value $(1,1,1,1)$ and the four Lyapunov 
exponents of the new hyperchaotic system (8) are $L E_{1}=0.0034131, L E_{2}=-0.98703$, $L E_{3}=-1.013$, and $\mathrm{LE}_{4}=-10.6689$. the Lyapunov exponents spectrum and Phase portraits of the system (8) are shown in Fig. 5 and Fig. 6 respectively.

So, we can obtain the Lyapunov dimension of the new hyperchaotic system (8), it is described as

$D_{L E}=j+\frac{1}{\left|L E_{j+1}\right|} \sum_{i=1}^{j} L E_{i}=3+\frac{L E_{1}+L E_{2}+L E_{3}}{\left|L E_{4}\right|}=3+\frac{0.0034131-0.98703-1.013}{|-10.6689|}=2.812856349$.

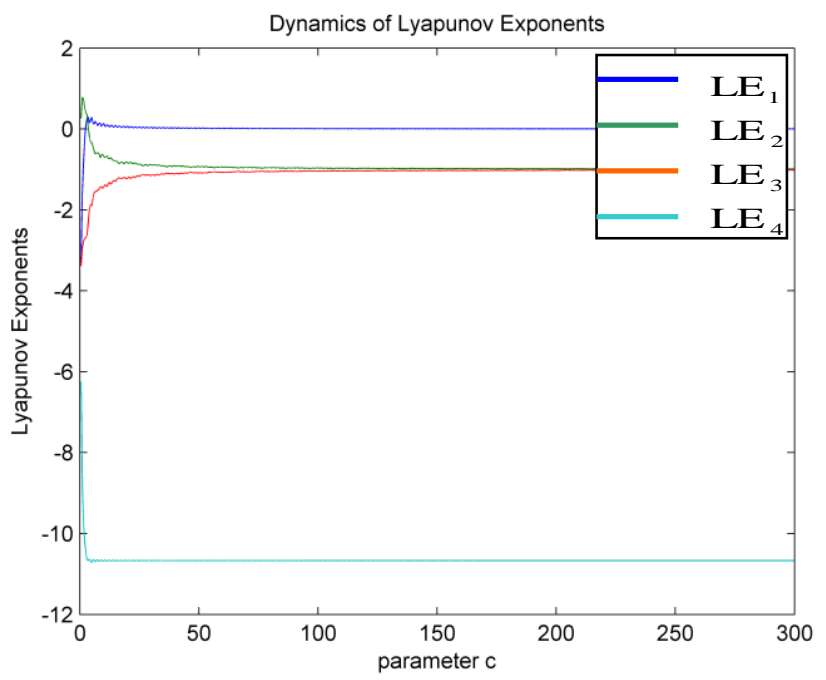

Fig. 5. Lyapunov exponents spectrum of a new hyperchaotic system versus parameter $\mathrm{c}$
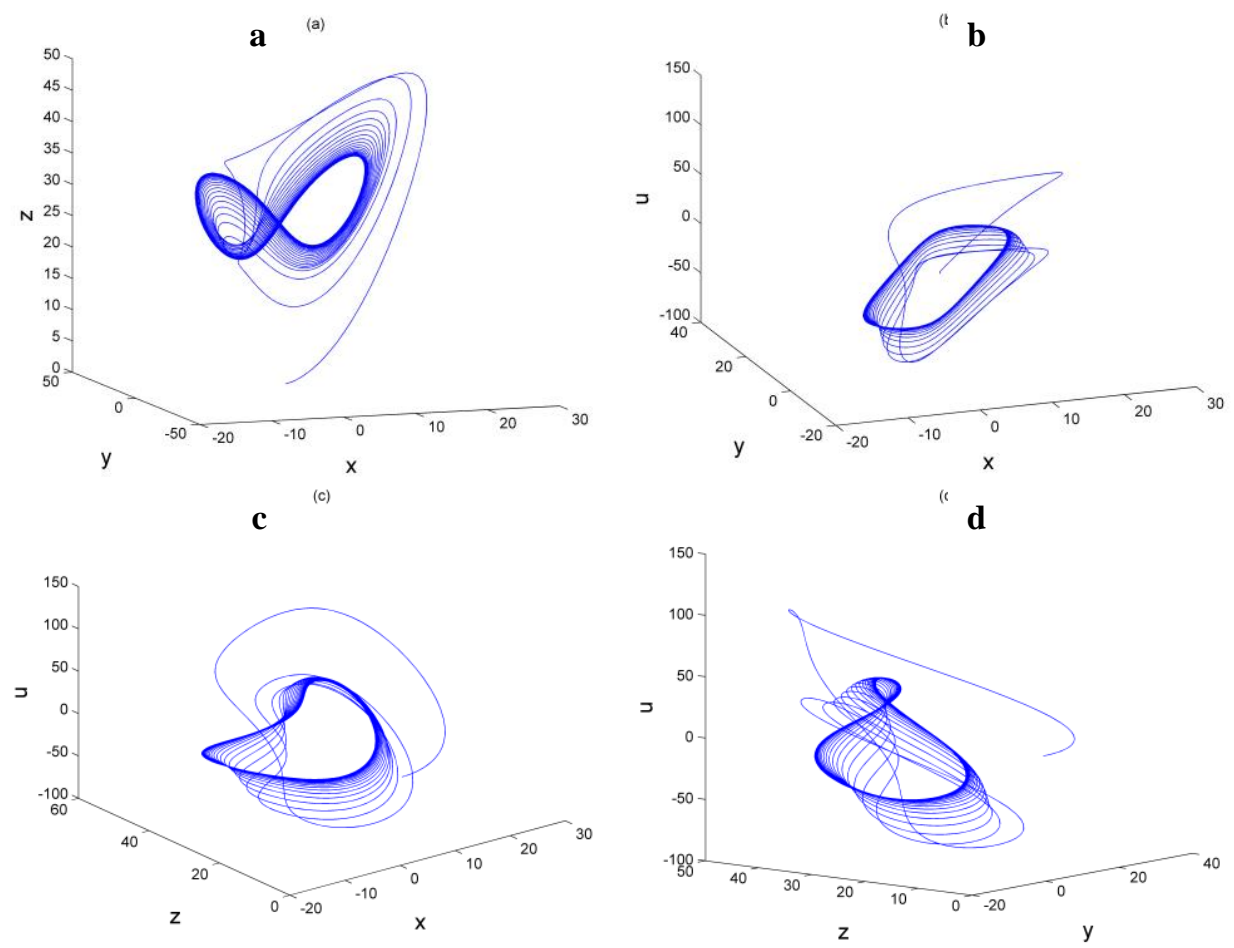

Fig. 6. Phase portraits of new hyperchaotic system in (a)the $x-y-z$ space;(b) the $x-y-u$ space, (c) the $x-z-u$ space, and(d) the $y-z-u$ space. 
Theorem 1: The solution of system (8) at the equilibrium point $O(0,0,0,0)$ when $a, b, d>0$ has the following cases:
1) Asymptotically stable if $c<0$,
2) Unstable if $c>0$,
3) Hopf bifurcation if $c=0$.

Proof: At the equilibrium point $O(0,0,0,0)$, system (8) are linearized, the Jacobian matrix defined as :

$$
J=\left[\begin{array}{cccc}
-a & a & 0 & 0 \\
c-z & 0 & -x & -1 \\
y & x & -b & 0 \\
z & d & x & 0
\end{array}\right]_{O(0,0,0,0)}=\left[\begin{array}{cccc}
-a & a & 0 & 0 \\
c & 0 & 0 & -1 \\
0 & 0 & -b & 0 \\
0 & d & 0 & 0
\end{array}\right]
$$

and its characteristic equation is :

$$
f(\lambda)=\lambda^{4}+(a+b) \lambda^{3}+(a b+d-a c) \lambda^{2}+(b d-a b c+a d) \lambda+a b d=0
$$

Solving equation (12) gives

$\lambda_{1}=-b$

and the following equation:

$$
f(\lambda)=\lambda^{3}+a \lambda^{2}+(d-a c) \lambda+a d=0
$$

Let

$$
A=a, B=d-a c, C=a d
$$

According to the Routh-Hurwitz condition, the real parts of roots $\lambda$ of (13) are negative if and only if

$$
a>0, \quad a d>0, \quad a(d-a c)-a d>0
$$

Implying $A, C>0$ since $a, d>0$ (given) and $A B-C>0$ it is possible under the condition $c<0$, therefore system (8) is asymptotically stable if $c<0$ while unstable if $c>0$. Finally, Hopf bifurcation if $c=0$, the proof is completed.

Due to the system is invariant under the transformation, so one only needs to consider the stability of any one of the both. The stability of the system (8) at equilibrium point $p_{1}$ is analyzed in this paper.

Theorem 2: The solution of system (8) at the equilibrium point $p_{1}$ is always unstable.

Proof: Now, to find Jacobian matrix at $p_{1}$ we need the following transformation under the linear transformation $(x, y, z, u) \rightarrow(X, Y, Z, U)$ :

$$
\left.\begin{array}{l}
x=X+\sqrt{-b d} \\
y=Y+\sqrt{-b d} \\
z=Z-d \\
u=U+\sqrt{-b d}(c+d)
\end{array}\right\}
$$

the system (8) becomes 


$$
\left.\begin{array}{l}
\dot{X}=a(Y-X) \\
\dot{Y}=(c+d) X-\sqrt{-b d} Z-U \\
\dot{Z}=\sqrt{-b d} X+\sqrt{-b d} Y-b Z \\
\dot{U}=-d X+d Y+\sqrt{-b d} Z
\end{array}\right\}
$$

The equilibrium point $p_{1}$ of the system (8) is switched to the new equilibrium point $O^{\prime}(0,0,0,0)$ of the system (15) under the linear transformation, The Jacobian matrix of the system $(15)$ at $O^{\prime}(0,0,0,0)$ is:

$$
J\left(O^{\prime}\right)=\left[\begin{array}{cccc}
-a & a & 0 & 0 \\
c+d & 0 & -\sqrt{-b d} & -1 \\
\sqrt{-b d} & \sqrt{-b d} & -b & 0 \\
-d & d & \sqrt{-b d} & 0
\end{array}\right]
$$

and the characteristic equation is :

$$
\lambda^{4}+A \lambda^{3}+B \lambda^{2}+C \lambda+D=0
$$

where

$$
\left.\begin{array}{l}
A=a+b \\
B=a b-b d-c a-a d+d \\
C=-(3 a b d+b c a) \\
D=-2 a b d
\end{array}\right\}
$$

Using Routh-Hurwitz criterion, the equation (17) has all roots with negative real parts if and only if the conditions are satisfied as follows

$$
\left.\begin{array}{l}
A>0 \\
A B-C>0 \\
(A B-C) C-A^{2} D>0 \\
D>0
\end{array}\right\}
$$

Since $D=-2 a b d$ and $\mathrm{a}, \mathrm{b}$ and $\mathrm{d}$ are positive parameters, consequently $D<0$ always therefore then one of Routh-Hurwitz conditions not satisfied, consequently the system (8) is unstable, the proof is completed.

Proposition 1: Equation (12) has purely imaginary roots if and only if $a, b, d>0$, $c=0=c_{0}$. In this case, the solutions of equation (12) are $\lambda_{1}=-b, \lambda_{2}=-a$, $\lambda_{3,4}= \pm i \sqrt{d}$.

Proof: First get one root $\lambda_{4}=-b$ from equation (12), then obtain cubic equation (equation 13). If $\lambda_{2,3}= \pm i w$ are the complex solutions and $\lambda_{1}$ the real solution of equation (12) then, from $\lambda_{1}+\lambda_{2}+\lambda_{3}=-a \Rightarrow \lambda_{1}=-a$. This easily leads to $a, b, d>0$, $c=0=c_{0}$ and $\lambda_{1}=-a, \lambda_{2,3}= \pm i \sqrt{d}, \lambda_{4}=-b$. 
In the following, we will prove that the system (8) displays a Hopf bifurcation at the point $O(0,0,0,0)$. For $c=c_{0}=0$, the point $o(0,0,0,0)$ loses its stability.

Theorem 3: If $c=0$, equation (12) has a negative solutions $\lambda_{4}=-b<0, \lambda_{1}=-a<0$ together with a pair of purely imaginary roots $\lambda_{2,3}= \pm i \sqrt{d}$ such that $\operatorname{Re}\left(\lambda_{c}^{\prime}\left(c_{0}\right)\right) \neq 0$, therefore the system (8) displays a Hopf bifurcation at the point $o(0,0,0,0)$.

Proof: If $c=0$ the equation (13) is transformed into

$(\lambda+a)\left(\lambda^{2}+d\right)=0$

with solutions $\lambda_{1}=-a, \lambda_{2,3}= \pm i \sqrt{d}$

$\lambda_{c}^{\prime}=\frac{a \lambda}{3 \lambda^{2}+2 a \lambda+d-c a}$

$\lambda_{c}^{\prime}\left(c_{0}\right)=\left.\frac{a \lambda}{3 \lambda^{2}+2 a \lambda+d-a c}\right|_{c=0}$

Substituting $\lambda_{2,3}= \pm i \sqrt{d}$, the real part and imaginary part of the $\lambda_{c}^{\prime}\left(c_{0}\right)$ respectively are:

$$
\begin{aligned}
& \operatorname{Re}\left(\lambda_{c}^{\prime}\left(c_{0}\right)\right)=\frac{2 a^{2} d}{(-2 d-a c)^{2}+4 a^{2} d} \neq 0, \\
& \operatorname{Im}\left(\lambda_{c}^{\prime}\left(c_{0}\right)\right)=\frac{a \sqrt{d}(-2 d-a c)}{(-2 d-a c)^{2}+4 a^{2} d} \neq 0 .
\end{aligned}
$$

Consequently, the system (8) displays a Hopf bifurcation at $O(0,0,0,0)$.

Corollary 1: System (8) at all equilibrium points $O, p_{1}, p_{2}$ has the following cases:

at least one root positive real part if $a<-b$, at least one root negative real part if $a>-b$,

either all roots have zero real parts, or there is a pair of roots whose real parts have opposite signs if $a=-b$.

Proof: By trace test (remark 3), we get $a_{11}+a_{22}+a_{33}+a_{44}=-a-b$ for each matrix (11) and (16) when, then at least one root has positive real part $-a-b>0 \Rightarrow a<-b$ and when, finally one root has negative real part, then at least $-a-b<0 \Rightarrow a>-b$ when $-a-b=0 \Rightarrow a=-b$ then either all roots have zero real parts, or there is a pair of roots whose real parts have opposite signs for all equilibrium points $O, p_{1}, p_{2}$.

Corollary 2: System (8) has, at least, one root with nonnegative real part at equilibrium point $O(0,0,0,0)$ if one of the following cases is satisfied:
(1) $a \leq-b$
(2) $a \leq \frac{-d}{b-c}$
(3) $a \leq \frac{-b d}{d-b c}$
(4) $a b d \leq 0$ 
Proof: By coefficient test (remark 1), when one coefficient of equation (12) $A, B, C, D \leq 0$, then, at least, one root has a nonnegative real part; consequently where
(1) $A=a+b$
if $A \leq 0$ then $a+b \leq 0$
$\Rightarrow a \leq-b$
(2) $B=a b+d-a c$
if $\quad B \leq 0$ then $a b+d-a c \leq 0 \Rightarrow a \leq \frac{-d}{b-c}$
(3) $C=b d-a b c+a d$ if $C \leq 0$ then $\quad b d-a b c+a d \leq 0 \Rightarrow a \leq \frac{-b d}{d-b c}$
(4) $D=a b d$
if $\quad D \leq 0$ then $a b d \leq 0$.

Corollary 3: System (8) has at least one root with nonnegative real part at equilibrium point $p_{1}$ if one of the following cases is satisfied:

$\left.\begin{array}{l}\text { (1) } a \leq-b \\ \text { (2) } a \leq \frac{d(b-1)}{b-c-d} \\ \text { (3) } c \geq-3 d \\ \text { (4) }-2 a b d \leq 0\end{array}\right\}$

Proof: By coefficient test (remark 1), when one coefficient of equation (17) $A, B, C, D \leq 0$, then, at least, one root has a nonnegative real part. consequently where
(1) $A=a+b$
if $A \leq 0$ then $a+b \leq 0$
$\Rightarrow \quad a \leq-b$

(2) $B=a b-b d-c a-a d+d$ if $\mathrm{B} \leq 0$ then $a b+d-a c \leq 0 \Rightarrow a \leq \frac{d(b-1)}{b-c-d}$

(3) $C=-3 a b d-a b c$ if $C \leq 0$ then $-3 a b d-a b c \leq 0 \Rightarrow c \geq-3 d$

(4) $D=-2 a b d \quad$ if $D \leq 0$ then $-2 a b d \leq 0$.

\section{5 - Illustrative Example:}

Example: Investigate the stability and Hopf bifurcation at the equilibrium point $O(0,0,0,0)$ of the following new hyperchaotic Pan system:

$\left.\begin{array}{l}\dot{x}=10(y-x) \\ \dot{y}=3 x-x z-u \\ \dot{z}=x y-5 z \\ \dot{u}=x z+y\end{array}\right\}$

Solution: $a=10, c=3, b=5, d=1$ and linearized system (22) about the equilibrium point $O(0,0,0,0)$ yield the following characteristic equations:

$\lambda^{4}+15 \lambda^{3}+19 \lambda^{2}-135 \lambda+50=0$

or $\lambda_{4}=-5$

$\lambda^{3}+10 \lambda^{2}-29 \lambda+10=0$

by using Routh-Hurwitz method on equation (24), we lead $A=10, C=10$ so $A, C>0$ and $A B=-290<C=10$ not satisfied Routh-Hurwitz conditions since $c=3>0$ (by theorem 1,(2)), the system (22) is unstable when $c>0$. 
but, if $c<0$ (by theorem 1, (1)), let $c=-2$ then yield the following characteristic equations:

$\mathrm{s} \lambda^{3}+10 \lambda^{2}+21 \lambda+10=0$

where satisfied Routh-Hurwitz conditions, consequently, the system (22) is asymptotically stable at origin when $c<0$.

If $\mathrm{c}=0$ then, yield the following characteristic equations:

$\lambda^{3}+10 \lambda^{2}+\lambda+10=0$

(satisfied Theorem 1(3)) the system (22) is bifurcation at $O(0,0,0,0)$. To justify these results we found the roots of equations (24), (25) and (26) the roots of equation ( 24) are $\lambda_{1}=2, \lambda_{2}=-6+\frac{\sqrt{164}}{2}, \lambda_{3}=-6-\frac{\sqrt{164}}{2}$.

While, the roots of equation (25) are $\lambda_{1}=-2, \lambda_{2}=-4+\frac{\sqrt{44}}{2}, \lambda_{3}=-4-\frac{\sqrt{44}}{2}$.

And the roots of equation (26) are $\lambda_{1}=-10, \lambda_{2}=-i, \lambda_{3}=+i$.

Consequently, the system (22) is asymptotically stable at equilibrium point $O(0,0,0,0)$ if $c<0$ and unstable if $c>0$ and Hopf bifurcation if $c=0$.

\section{6- Conclusion:}

This paper presents a new four dimensional hyperchaotic system, which is called a new hyperchaotic Pan system. This new hyperchaotic system is different from the hyperchaotic Lorenz system, hyperchaotic Lü system, another hyperchaotic Lü system as well as hyperchaotic system which was proposed by Pan in Ref [5]. Since, the new hyperchaotic system has more complex dynamical behaviors than the normal chaotic systems, it is believed that the system will have broad applications in various chaosbased information systems. 


\section{REFERENCES}

[1] Borrelli R.L., Coleman C.S. (1998), Differential Equations, New York, John Wiley and Sons, Inc.

[2] Chen A., Lu J., Lü J., Yu S., (2006), Generating Hyperchaotic Lü Attractor Via State Feedback Control, Journal of Science Direct, Physica A, No. 364, pp. 103110.

[3] Jia H., Chen Z., Wu W., (2009), A New Hyper-Chaotic Lü Attractor and Its Local Bifurcation Analysis, International Workshop on Chaos-Fractals Theories and Applications pp. 231-235.

[4] Pan L., Xu D., Zhou W., (2010), Controlling a Novel Chaotic Attractor Using Linear Feedback, Journal of Information and Computing Science, Vol. 5, No. 2, pp. 117-124.

[5] Pan L., Zhou W., Zhou L., Sun K., (2011), Chaos Synchronization Between Two Different Fractional-Order Hyperchaotic Systems, Journal of Science Direct, Communications Nonlinear Science and Numerical Simulation, Vol. 16, pp. 2628-2640.

[6] Wang G., Zhang X., Zheng Y., Li Y., (2006), A New Modified Hyperchaotic Lü System, Journal of Science Direct, Physica A No. 371, pp. 260-272.

[7] Wang G., Zhen Y., Li Y., (2006), Controlling Lorenz System to Hyperchaotic, Proceedings of the 3nd International Conference on impulsive Dynamic System and Applications, pp. 1543-1545.

[8] Wang X., Wang M., (2008), A Hyperchaos Generated Form Lorenz System, Journal of Science Direct, Physica A, No. 387, pp. 3751-3758.

[9] Xue W., Mu J., Jia H., (2011), A Novel One Equilibrium Hyper-Chaotic System and Bifurcation Analysis, International Workshop on Chaos- Fractals Theories and Applications, pp. 197-201.

[10] Xu J., Cai G., Zheng S., (2009), A Novel Hyperchaotic System and Its Control, Journal of Uncertain system, Vol. 3, No. 2, pp. 137-144. 